Daniela BarReto Fragugla

QUental DINIZ1

Danella De Batista Depes ${ }^{2}$

ana Maria Gomes Pereira' ${ }^{1}$

Simone DenISE DAVID ${ }^{3}$

UMBERTO GAZI LIPPI ${ }^{4}$

Fausto Farah Baracat ${ }^{5}$

Reginaldo Guedes CoelHo Lopes $^{6}$

\section{Avaliação da dor em histeroscopia ambulatorial: comparação entre duas técnicas}

\author{
Pain evaluation in office bysteroscopy: comparison of two techniques
}

Palavras-chave

Histeroscopia/métodos Medição da dor

Cloreto de sódio

Assistência ambulatorial

Keywords

Hysteroscopy/methods Measurement of pain Sodium chloride Ambulatory care

\section{Resumo}

OBJETIVO: comparar a dor referida pelas pacientes submetidas à histeroscopia pela técnica convencional com gás carbônico $\left(\mathrm{CO}_{2}\right)$ e a vaginohisteroscopia com soro fisiológico (SF 0,9\%). MÉTODOS: estudo prospectivo de coorte, realizado em um serviço de histeroscopia ambulatorial. Foram incluídas 117 pacientes com indicação para realizarem o exame, alocadas aleatoriamente em dois grupos. Todas responderam a um questionário epidemiológico e quantificaram a dor esperada antes do exame e sentida após seu término em uma escala verbal de dor de 0 a 10. Para a técnica convencional, foram utilizados espéculo, tração do colo, inserção de ótica de $30^{\circ}$ e camisa diagnóstica com diâmetro total $5 \mathrm{~mm}$. A cavidade foi distendida com $\mathrm{CO}_{2}$ sob pressão de $100 \mathrm{mmHg}$ controlada por histeroinsuflador e a biópsia realizada com cureta de Novak. A vaginoscopia foi realizada sem toque, por distensão da vagina com líquido, visualização direta do colo e introdução de ótica com duas camisas de fluxo contínuo com canal acessório de perfil ovalado, totalizando também $5 \mathrm{~mm}$ de diâmetro para o conjunto. Foi utilizado SF 0,9\% como meio de distensão e a pressão, definida como a necessária para adequada visualização do canal e da cavidade com pressurizador pneumático externo. A biópsia foi realizada de forma dirigida com pinça endoscópica. Foram calculados média e desvio padrão para as variáveis quantitativas e frequência para as qualitativas. O teste t de Student foi utilizado para comparar médias e o teste do qui-quadrado ou exato de Fischer (quando $n<5$ ), para análise categórica usando o SPSS 15.0. O estudo foi desenhado para 95\% de poder do teste com significância se $\mathrm{p}<0,05$. RESULTADOS: os grupos foram similares quanto a: idade, paridade, cirurgia uterina prévia, estado menopausal e necessidade de biópsia. No grupo vaginoscopia, comparado ao da técnica convencional, houve menor dificuldade técnica $(5,1$ versus $17,2 \%, p=0,03)$, maior taxa de exames considerados satisfatórios $(98,3$ versus $89,7 \%, p=0,04)$ e menor índice de dor $(4,8$ versus 6,$1 ; p=0,01)$, com diferença mais evidente em comparação a pacientes que nunca haviam tido um parto normal prévio $(4,9$ versus 7,1 ; $p=0,0001$ ). Ao estratificar a escala de dor em leve (0-4), moderada (5-7) ou intensa (8-10) a técnica por vaginoscopia foi associada à redução de 52\% na frequência da dor intensa ( $p=0,005)$. CONCLUSÕES: A vaginohisteroscopia mostrou-se um procedimento menos doloroso do que a técnica com espéculo e $\mathrm{CO}_{2}$ independentemente de idade, menopausa ou paridade, com resultados mais satisfatórios e menor dificuldade técnica.

\section{Abstract}

PURPOSE: to compare the pain reported by patients submitted to hysteroscopy by the standard technique with carbon dioxide $\left(\mathrm{CO}_{2}\right)$ and to vaginal hysteroscopy with physiological saline $(0.9 \% \mathrm{NaCl})$. METHODS: this was a prospective cohort study conducted at an ambulatory hysteroscopy service. A total of 117 patients with indication for the exam were included, being randomly assigned to one of the groups. All patients answered an epidemiological questionnaire and scored the pain expected before the exam and that felt after the end of the procedure on a verbal pain scale from $\mathrm{O}$ to 10. A speculum, traction of the cervix, insertion of a $30^{\circ}$ light source and a diagnostic shirt with a total diameter of $5 \mathrm{~mm}$ were used for the standard technique. The cavity was distended with $\mathrm{CO}_{2}$ under a pressure of $100 \mathrm{mmHg}$ controlled with
Correspondência: Daniela Barreto Fraguglia Quental Diniz Hospital do Servidor Público Estadual Avenida lbirapuera, 981 - Vila Clementino CEP 04029-000 - São Paulo (SP), Brasil E-mail: dradanieladiniz@hotmail.com Fone/fax: (11) 5088-8085

Recebido

20/11/09

Aceito com modificações 20/12/09
Setor de Endoscopia Ginecológica do Serviço de Ginecologia e Obstetrícia do Hospital do Servidor Público Estadual de São Paulo "Francisco Morato de Oliveira" - HSPE-FMO - São Paulo (SP), Brasil.

'Médicas assistentes do Serviço de Ginecologia e Obstetrícia do Hospital do Servidor Público Estadual "Francisco Morato de Oliveira" - HSPE-FMO - São Paulo (SP), Brasil; Pós-graduandas (Mestrado) em Ciências da Saúde do Instituto de Assistência Médica ao Servidor Público Estadual - São Paulo (SP), Brasil.

2 Encarregada do Setor de Endoscopia Ginecológica do Serviço de Ginecologia e Obstetrícia do Hospital do Servidor Público Estadual "Francisco Morato de Oliveira" - HSPE-FMO - São Paulo (SP), Brasil.

"Médica assistente do Serviço de Ginecologia e Obstetrícia do Hospital do Servidor Público Estadual "Francisco Morato de Oliveira" - HSPE-FMO - São Paulo (SP), Brasil.

${ }^{4}$ Médico assistente do Serviço de Ginecologia e Obstetrícia do Hospital do Servidor Público Estadual "Francisco Morato de Oliveira" - HSPE-FMO - São Paulo (SP), Brasil.

${ }^{5}$ Chefe da seção de Ginecologia do Hospital do Servidor Público Estadual "Francisco Morato de Oliveira" - HSPE-FMO - São Paulo (SP), Brasil.

•Diretor do Serviço de Ginecologia e Obstetrícia do Hospital do Servidor Público Estadual "Francisco Morato de Oliveira" - HSPEFMO - São Paulo (SP), Brasil.

Os autores declaram não terem recebido auxílios ou financiamentos, não havendo assim conflito de interesses. 
a hysteroflator, and a biopsy was obtained with a Novak curette. Vaginoscopy was performed without a touch by distention of the vagina with fluid, direct visualization of the cervix and introduction of the light source with two continuous-flow shirts, with an accessory channel with an oval profile, the whole set measuring $5 \mathrm{~mm}$ in diameter. The medium distention was $0.9 \% \mathrm{NaCl}$ and the pressure used was that considered to be necessary for an adequate visualization of the canal and of the cavity with an external pneumatic pressurizer. The biopsy was obtained in a directed manner using an endoscopic clamp. The mean and standard deviation were calculated for the quantitative variables and the frequency was calculated for the qualitative variables. The Student's ftest was used to compare the means, and the chi-square or exact Fisher test was used (when $n<5$ ) for the categorical analysis using the SPSS 15.0 software. The study was designed for a $95 \%$ test power, with the level of significance set at $\mathrm{p}<0.05$. RESULTS: the groups were similar regarding age, parity, previous uterine surgeries, menopausal status, and the need for a biopsy. In comparison to the group submitted to the standard technique, the vaginoscopy group involved a lower technical difficulty (5.1 versus $17.2 \%, p=0.03)$, a higher rate of exams considered to be satisfactory $(98.3$ versus $89.7 \%, p=0.04)$ and a lower pain index $(4.8$ versus $6.1 ; p=0.01)$, as the difference were more evident when patients who never had a previous normal delivery were compared (4.9 versus $7.1 ; p=0.0001)$. When the pain scale was stratified as mild (0-4), moderate (5-7) or intense (8-10), the vaginoscopy technique was found to be associated with a $52 \%$ reduction of the frequency of intense pain ( $p=0.005)$. CONCLUSIONS: vaginohysteroscopy was proved to be a less painful procedure than the technique based on the use of a speculum and $\mathrm{CO}_{2}$, regardless of age, menopause or parity, with more satisfactory results and lower technical difficulty.

\section{Introdução}

A vídeo-histeroscopia ambulatorial tem importante papel na propedêutica ginecológica por ser simples, segura e de boa acurácia ${ }^{1}$. Diversos fatores colaboraram para sua disseminação, dentre os quais destaca-se o uso de óticas com sistema de lentes mais avançados, a utilização de câmera acoplada e a inclinação de $30^{\circ}$ que permite uma visão panorâmica com um leve giro no seu eixo. Mas o que mais contribuiu para a exequibilidade do exame foi a redução do diâmetro das óticas, permitindo sua introdução em canais endocervicais estreitos sem trauma ou necessidade de dilatação ${ }^{2,3}$.

Apesar dos avanços, a histeroscopia ambulatorial ainda encontra dificuldades. A visualização da cavidade e do canal endocervical torna-se ineficiente ou muito prejudicada quando o sangramento está presente, pois o meio de distensão comumente utilizado, gás carbônico $\left(\mathrm{CO}_{2}\right)$, produz bolhas e não consegue remover fragmentos de mucosa que mantêm suja a extremidade de contato da ótica ${ }^{4,5}$. Outra limitação é a pobre correlação histológica obtida quando são comparados os achados visuais de lesões focais (sensibilidade de 98\%) ao exame anatomopatológico de endométrio obtido por biópsias às cegas orientadas (sensibilidade de 65\%) 2,6,7 $^{2}$ Por fim, mas primordial, é a dor que o exame pode trazer, limitando ou interrompendo a avaliação precisa da cavidade e impedindo que possa ser repetido para futuros controles, já que será associado a desconforto e medo ${ }^{8}$.

A dor e o desconforto na histeroscopia diagnóstica podem ser inicialmente ocasionados pela introdução do espéculo, levando à distensão das paredes vaginais, pela limpeza do colo, pelo pinçamento e tração com a pinça de Pozzi, pela dilatação do canal endocervical e, por último, pela passagem pelo orifício interno (local de maior concentração de terminações nervosas e menor diâmetro). A distensão da cavidade e o estiramento da mucosa endometrial podem aumentar a dor. No final, após retirado o instrumental, é realizada a biópsia endometrial, que pode ser mais dolorosa do que o procedimento em $\mathrm{si}^{7,9}$. A dor hipogástrica tipo cólica referida durante o exame se deve à distensão da cavidade e, quando a pressão leva a abertura dos óstios tubários, pode haver dor escapular pela presença de $\mathrm{CO}_{2}$ livre na cavidade peritoneal e irritação frênica ${ }^{10}$. Outra possibilidade é a resposta vagal, com incidência de $0,5 \%$, causada por estímulo parassimpático na passagem pelo canal cervical ou distensão da cavidade, caracterizada por hipotensão, bradicardia, náuseas, sudorese e tontura, geralmente autolimitada ${ }^{11}$.

Apesar de alguns estudos mostrarem que $70 \%$ das pacientes podem ter efeitos adversos, o exame é realizado de forma satisfatória em 90 a 100\% dos casos com taxa de aceitação de 83 a $99 \%^{7}$. As tentativas de redução da dor por meio de anestesia tópica foram mal sucedidas, por vezes sendo mais doloridas do que o procedimento em si, e portanto só indicada quando houver dilatação mecânica do canal ${ }^{12}$. Analgésicos, misoprostol e anti-inflamatórios também não se mostraram eficazes durante o exame, apenas melhorando o desconforto após 30 a 60 minutos ${ }^{7,13-17}$.

Uma nova abordagem foi desenvolvida para se realizar a histeroscopia ambulatorial sem a utilização de espéculo e de pinça de Pozzi, empregando-se o soro fisiológico (SF 0,9\%) como meio de distensão ${ }^{18}$. Utilizamse duas camisas de perfil ovalado com fluxo contínuo que permitem melhor visualização da cavidade. $\mathrm{O}$ colo e o orifício externo são visualizados com distensão da vagina pelo soro, e a introdução do histeroscópio é mais fisiológica, girando em $90^{\circ}$ o conjunto para ultrapassar horizontalmente o orifício interno ${ }^{3}$. Quando se alcança a cavidade, é possível usar pinça ou tesoura para a realização de biópsias dirigidas, retirada de corpos estranhos ou pequenas cirurgias ${ }^{19}$.

Estudos recentes demonstraram significativa redução da dor referida com a utilização dessa técnica de histeroscopia, com índices de dor variando de 0,5 a 6,0 $0^{1,7,10,19}$. O objetivo do presente estudo foi quantificar e avaliar a dor referida pelas pacientes quando utilizadas as técnicas convencional com $\mathrm{CO}_{2}$ e vaginohisteroscopia com SF $0,9 \%$. 


\section{Métodos}

Estudo prospectivo de coorte, controlado, intervencionista realizado no hospital-escola público Hospital do Servidor Público Estadual "Francisco Morato Oliveira", Instituto de Assistência Médica ao Servidor Público Estadual, São Paulo (SP), Brasil, entre abril de 2005 e outubro de 2006. Foram incluídas as pacientes com indicação de realização de histeroscopia diagnóstica que concordaram em participar e assinaram o termo de consentimento livre e informado. Esse trabalho recebeu a aprovação do Comitê de Ética em Pesquisa da instituição.

A amostragem foi aleatória, incluindo todas as pacientes atendidas consecutivamente pela equipe do Setor de Endoscopia Ginecológica durante o período do estudo, alocadas nos diferentes grupos por alternância de técnicas. $\mathrm{O}$ estudo incluiu 117 mulheres distribuídas em dois grupos: $59(50,4 \%)$ no Grupo 1 (técnica por vaginoscopia) e 58 $(49,6 \%)$ no Grupo 2 (técnica convencional). O exame foi realizado no ambulatório especializado de histeroscopia sem anestesia ou analgesia por duas médicas ginecologistas com habilitação em endoscopia ginecológica e em regime de revezamento.

Os critérios de inclusão foram: idade superior a 18 anos, solicitação médica de histeroscopia independentemente da indicação do procedimento. Foram excluídas as pacientes que não concordaram em participar do estudo ou apresentavam contraindicações ao exame (gestação, cervicites, sangramento genital e doença inflamatória pélvica aguda) ${ }^{11}$.

A técnica por vaginoscopia consistiu no uso de ótica de $2,9 \mathrm{~mm} 30^{\circ} \mathrm{com}$ duas camisas para fluxo contínuo, compondo um total de $5 \mathrm{~mm}$ com perfil ovalado (Histeroscópio de Bettocchi, Karl Storz ${ }^{\circledR}$, Alemanha). A visualização do colo sem espéculo ou pinça de Pozzi ocorreu por distensão vaginal e permitiu a progressão no canal cervical e cavidade utilizando-se soro fisiológico $(\mathrm{SF} 0,9 \%)$ em temperatura ambiente com pressão determinada por gravidade e enchimento de manguito de pressão ao redor do frasco ${ }^{2}$, sendo suficiente para uma visualização adequada do canal cervical e da cavidade uterina. As biópsias dirigidas foram realizadas por uma pinça introduzida pelo canal acessório quando encontradas anormalidades no endométrio.

A técnica convencional consistiu no uso de espéculo e tração cervical por pinçamento com pinça de Pozzi no lábio anterior para introdução de ótica de $4 \mathrm{~mm} 30^{\circ}$, com camisa diagnóstica circular de $5 \mathrm{~mm}$ do mesmo fabricante. A distensão foi realizada com $\mathrm{CO}_{2}$ com pressão controlada por histeroinsuflador em $100 \mathrm{mmHg}^{3}$, e as biópsias orientadas realizadas quando necessárias após a remoção da ótica, com cureta de Novak acoplada a seringa de $20 \mathrm{~mL}$ sob sucção ${ }^{20}$. Os exames foram considerados satisfatórios quando canal endocervical e cavidade uterina foram visualizados com clareza, permitindo elaborar hipóteses diagnósticas.

Todas as pacientes responderam a um questionário, aplicado pela equipe médica na admissão, com o objetivo de avaliar possíveis diferenças de conhecimento e expectativa a respeito do exame. Foi utilizada uma escala analógica verbal de dor (EVD) $)^{21,22}$ quantitativa discreta, variando de zero (equivalente a nenhuma dor) a dez (a pior dor já experimentada). As pacientes quantificaram a dor que esperavam sentir antes do exame, e a que realmente sentiram, imediatamente após o término do exame. Seguindo artigos recentes, que demonstram não haver relevância na dor após o exame, não foram feitas reavaliações $\operatorname{tardias}^{23}$.

As variáveis incluídas foram idade, número de gestações, paridade, EVD antes e após o exame, tempo pósmenopausa, histórico de cirurgia uterina prévia (incluindo cesárea, curetagem, conização e histeroscopia cirúrgica), necessidade de biópsia, presença de dificuldade técnica, sucesso no procedimento e concordância da paciente em se submeter novamente ao exame caso fosse necessário. A dor após o exame, principal variável do estudo, foi estratificada em três categorias: de 0 a 4 (dor mínima), 5 a 7 (dor moderada) e 8 a 10 (dor intensa) ${ }^{4}$.

O cálculo amostral foi feito para nível de significância a $5 \%(p=0,05)$ com poder do teste de $95 \%$ para comparar duas médias, de forma a detectar qualquer mudança de pelo menos dois pontos em dez na escala de dor (critério capaz de promover mudança de categoria $)^{4}$. $\mathrm{O}$ valor necessário foi de 58 pacientes em cada grupo.

Todas as informações e resultados foram analisados utilizando-se o software estatístico SPSS 15.0 para Windows, com cálculo descritivo de média e desvio padrão para variáveis quantitativas e de frequência para variáveis qualitativas. A análise categórica foi feita por meio do teste do qui-quadrado ou Exato de Fischer (quando variável menor do que 5) e a comparação de médias com teste $t$ de Student bicaudado para amostras não-pareadas. A significância estatística foi estabelecida como $\mathrm{p}<0,05$.

\section{Resultados}

O estudo incluiu 117 mulheres com características listadas na Tabela 1. Os grupos foram homogêneos e similares quanto à idade, menopausa, histórico obstétrico e ginecológico, necessidade de biópsia e concordância em repetir o exame se necessário.

A técnica convencional foi significativamente mais difícil de se realizar do que a vaginoscopia (17,2 versus $5,1 \%$, respectivamente) com resultados menos satisfatórios $(89,7$ versus $98,3 \%)$ devido à presença de sangramento ou de estenose de orifício interno. (Tabela 1). 
Tabela 1 - Distribuição das pacientes submetidas à histeroscopia por vaginoscopia ou pela técnica convencional de acordo com características populacionais, variáveis do procedimento e aceitação em repetir 0 exame

\begin{tabular}{|c|c|c|c|}
\hline & Vaginoscopia $(n=59)$ & Convencional $(n=58)$ & Valor de $\mathrm{p}$ \\
\hline Idade [média (faixa de variação)] & $53,2[29-81]$ & $50,9[21-86]$ & $0,3^{\dagger}$ \\
\hline \multicolumn{4}{|l|}{ Antecedentes obstétricos } \\
\hline Nuliparas & $4[6,9]$ & $2[3,4]$ & $0,6^{*}$ \\
\hline Gestações§ [faixa de variação] & $3,52[1-13]$ & $2,81[1-13]$ & $0,2^{\dagger}$ \\
\hline Partos§ [média (faixa de variação)] & $3,00[0-10]$ & $2,37[1-5]$ & $0,4^{\dagger}$ \\
\hline Pós-menopausa & $29(50)$ & $19(33,3)$ & 0,07 \\
\hline Necessidade de biópsia & $41(69,5)$ & $36(62,1)$ & 0,3 \\
\hline Dificuldade técnica & $3(5,1)$ & $10(17,2)$ & 0,03 \\
\hline Exame satisfatório & $58(98,3)$ & $52(89,7)$ & 0,04 \\
\hline Paciente aceita repetir o exame & $53(89,8)$ & $53(91,4)$ & 0,9 \\
\hline
\end{tabular}

Valores expressos em n (\%), a não ser se indicado de outra forma; análise estatística: $\chi^{2}$.

*Teste exato de Fischer; †'teste $t$ de Student; ${ }^{\S}$ excluíndo as nulíparas (Grupo Vaginoscopia n=55, Grupo Convencional n=56).

Tabela 2 - Comparação da dor nos grupos de histeroscopia por vaginoscopia ou técnica convencional após estratificação dos índices de dor em subgrupos: dor leve, moderada e intensa

\begin{tabular}{|c|c|c|c|c|c|c|c|c|}
\hline \multirow{2}{*}{ Grupos } & \multicolumn{2}{|c|}{ Dor leve (0-4) } & \multicolumn{2}{|c|}{ Dor moderada (5-7) } & \multicolumn{2}{|c|}{ Dor intensa $(8-10)$} & \multicolumn{2}{|c|}{ Total } \\
\hline & $\mathrm{n}$ & $\%$ & $\mathrm{n}$ & $\%$ & $\mathrm{n}$ & $\%$ & n & $\%$ \\
\hline Vaginoscopia & 28 & $47,5^{*}$ & 19 & 32,2 & 12 & $20,3^{*}$ & 59 & 100 \\
\hline Convencional & 15 & $25,8^{*}$ & 19 & 32,8 & 24 & $41,4^{*}$ & 58 & 100 \\
\hline
\end{tabular}

Análise estatística $\chi^{2}$ bicaudal.

${ }^{*} \mathrm{p}=0,005$; risco relativo $=0,48(\mathrm{IC}=0,28-0,83)$.

Antes da histeroscopia, ambos os grupos mostraram-se homogêneos quanto às expectativas referentes ao exame. A maioria das pacientes de ambos os grupos nunca havia feito o exame $(79,5 \%)$ nem sabia como seria feito $(68,4 \%)$, mas conheciam seu nome $(67,5 \%)$ e sua indicação $(53 \%)$. Acreditavam também que sua realização seria importante (96,6\%). A expectativa de dor (calculada a média dos índices de dor) não diferia nos dois grupos, sendo de 6,5 no grupo no qual foi empregada a nova técnica e 6,0 no controle $(\mathrm{p}=0,21)$.

Após o exame, a média em cada visual de dor mostrou uma grande e significativa diferença havendo redução de 6,1 quando usada a técnica convencional para 4,8 a favor da técnica de vaginoscopia, com p=0,01 (IC -2,3 a -0,2) pelo teste $t$ de Student.

Os antecedentes obstétricos demonstraram influência na percepção da dor. As pacientes submetidas ao exame pela técnica convencional e que tinham tido pelo menos um parto normal anterior apresentaram média de dor significativamente menor durante o exame em comparação às nulíparas ou àquelas somente com cesariana anterior (respectivamente 5,5 e 7,1, p=0,03). As pacientes submetidas ao exame por vaginoscopia relataram dor menos intensa do que as do grupo convencional quando havia antecedente de parto vaginal (média EVD 4,8 versus 5,5 , respectivamente; $\mathrm{p}=0,03$ ) e essa diferença ficou ainda mais evidente quando selecionadas só as pacientes que não tiveram parto normal prévio (respectivamente 4,9 versus $7,1, \mathrm{p}=0,0001)$. Destaca-se dão fato de a média de dor no grupo de vaginoscopia sem nenhum parto normal prévio (média EVD 4,9) ser também menor do que a média de dor do grupo da técnica convencional com histórico de parto normal (média EVD 5,5). No grupo da vaginoscopia, a média da dor se manteve baixa independentemente do antecedente de parto vaginal (4,8 quando presente e 4,9 quando ausente, com diferença não-significativa).

A dor foi estratificada em três níveis de intensidade ${ }^{5}$, sendo categorizada como dor leve se o índice da escala verbal de dor ficasse entre 0 a 4 , moderada entre 5 e 7 , e dor intensa quando maior ou igual a 8 . O grupo da técnica da vaginoscopia manifestou dor leve em $47,5 \%$ dos casos e leve ou moderada em 79,7\%; o grupo da antiga técnica apresentou dor intensa em $41,4 \%$ dos casos. Essa nítida diferença na distribuição é apresentada na Tabela 2 e demonstra significativa redução de $52 \%$ para a dor intensa no grupo que realizou a histeroscopia pela técnica proposta.

Outros fatores que poderiam modificar a EVD, como a pós-menopausa, (37 pacientes, 31,6\%); submissão prévia à histeroscopia (24 pacientes, 20,5\%) e realização de biópsia (77 pacientes, $65 \%$,) não mostraram influência sobre os resultados. 


\section{Discussão}

A histeroscopia, desde a década de 1980, tem conquistado cada vez mais espaço na investigação das afecções intrauterinas e endocervicais não somente por ser método de excelência para a visualização da cavidade e canal cervical, mas também por ter se tornado cada vez mais disponível e acessível ${ }^{1}$. Em revisão da literatura que incluiu 10.232 pacientes submetidas ao exame, foram encontradas taxas de aceitação de 83 a $99 \%^{7}$.

Sem dúvida, a redução no diâmetro das óticas foi o fator que permitiu que o procedimento saísse do centro cirúrgico e passasse ao regime ambulatorial, reduzindo $\operatorname{dor}^{24}$ e reflexo vagal ${ }^{1,24}$. A introdução da nova técnica de abordagem descrita nesse estudo é mais um importante passo na história da endoscopia ginecológica ${ }^{2}$. A utilização do SF 0,9\% como meio de distensão permite fluxo contínuo e oferece maior acurácia para a detecção de pequenas lesões e para a avaliação do sangramento uterino anormal ${ }^{5,25}$, além de permitir exames mais rápidos e com melhor aceitação pelas pacientes ${ }^{26,27}$. A técnica não difere apenas no meio de distensão, mas em um conjunto de práticas; como a facilidade de se dispor de um frasco de SF $0,9 \%$ em comparação à necessidade de $\mathrm{CO}_{2}$ e histeroinsuflador, e pelo fato de permitir realizar o exame sem distensão vaginal pelo espéculo e sem o pinçamento do colo com a pinça de Pozzi ${ }^{28}$. A taxa de pacientes que referem ausência de dor chega a 98,9\% quando avaliada a vaginoscopia até a introdução da ótica no orifício externo ${ }^{2,29}$ e a taxa de sucesso de $90^{7}$ a $99 \%{ }^{23}$.

Outra vantagem dessa técnica é melhorar a acurácia do exame que antes era determinada principalmente pela capacidade do examinador de observar a lesão, já que se tratava de exame fundamentalmente visual ${ }^{2}$. Com as novas camisas de exame com canal acessório é possível introduzir microinstrumentos, como pinças, tesouras, ponteiras inclusive com corrente bipolar, não só para realizar biópsias dirigidas em lesões focais como também para tratar algumas afecções intrauterinas benignas ${ }^{3,8}$. A revisão de 4.863 casos de histeroscopia ambulatorial, incluindo cirurgias realizadas com microinstrumentos mecânicos, mostrou grande taxa de sucesso ${ }^{2,30}$ com desconforto ausente em 71,9 a 93,5\% das pacientes ${ }^{18}$. Esse procedimento, conhecido como "see and treat", pode ser empregado para remoção de pequenos pólipos, inserção de dispositivos tubários contraceptivos, lise de sinéquias, reposicionamento de dispositivo intrauterino. É feito de forma rápida e indolor, pois a inervação uterina começa no miométrio, o que permite manipulação do endométrio e tecido conjuntivo. Porém, como toda técnica, depende da experiência do histeroscopista ${ }^{3}$.

Os valores dos índices de dor mostrados têm resultados conflitantes, mas a dor é semrpe classificada como leve ou moderada. Dentre os estudos que se assemelham ao nosso mostrando dor moderada ${ }^{5,31}$, destacam-se um estudo realizado no Brasil, em 2008, com média de dor de $6,0^{19}$ e outros dois mais recentes com população indiana com média de 4,1 a 5,932-34. A maioria dos trabalhos são europeus e descrevem média de dor leve variando de 1,6 a $4^{4,27,28,30,35-37}$. Alguns descrevem ausência de dor em 78 a $90 \%$ das pacientes ${ }^{1,18}$ ou média de dor de 0,5 em uma escala de 0 a $10^{10}$. Esses dados são discrepantes quando comparados aos do presente trabalho. Isto pode ser devido à grande experiência dos serviços citados aliada à maior prevalência de partos normais nesses países em relação à população brasileira. Outros estudos, no entanto, não mostram diferenças na avaliação da $\operatorname{dor}^{5,23,25}$.

Histórico de parto normal como variável capaz de determinar redução da dor não encontra consenso na literatura, aparecendo em alguns estudos como nãodeterminante ${ }^{7,31}$, mas mostrando grande impacto na sua redução em um ensaio clínico com análise multivariada ${ }^{35}$. No Brasil, país com grande taxa de cesarianas no sistema de saúde complementar, o uso de técnica capaz de reduzir a dor independentemente do histórico obstétrico e da via de parto, como ocorre com a técnica da vaginoscopia, é muito bem-vindo e tem grande aplicabilidade.

É plausível considerar que um maior grau de aceitação do exame poderia ser obtido oferecendo à paciente uma explicação detalhada do método, reduzindo assim sua ansiedade, como demonstrado na literatura ${ }^{30,34}$. Este estudo, porém, mostrou que as pacientes informadas pelos médico a respeito do procedimento não experimentaram nenhum alívio em seu desconforto, e aquelas que já haviam sido submetidas à histeroscopia anteriormente $\mathrm{e}$ conheciam todas as suas etapas referiram dor semelhante àquelas referidas pelas pacientes que nunca haviam sido submetidas ao exame.

Apesar de haver diversas evidências de que o climatério leva à atrofia genital e pode dificultar a realização do exame $^{3}$, que a realização da biópsia guiada ou orientada ${ }^{38}$ por cureta ou Pipelle pode ser tão dolorosa quanto ou até mais desconfortável do que o exame em $\mathrm{si}^{\mathrm{i}}{ }^{7,9}$, os achados deste estudo não confirmam tais afirmações. Essa limitação se deve provavelmente ao fato de que o cálculo amostral foi feito baseado em avaliação de dor, incluindo todos os exames, e, como apenas um terço estava na pós-menopausa e em dois terços dos casos se realizou biópsia, a amostra não foi grande o suficiente para permitir conclusões. 
1. Cicinelli E, Rossi AC, Marinaccio M, Matteo M, Saliani N, Tinelli R. Predictive factors for pain experienced at office fluid minihysteroscopy. J Minim Invasive Gynecol. 2007; 14(4):485-8.

2. Bettocchi S, Nappi L, Ceci O, Selvaggi L. What does 'diagnostic hysteroscopy' mean today? The role of the new techniques. Curr Opin Obstet Gynecol. 2003;15(4):303-8.

3. Rullo S, Sorrenti G, Marziali M, Ermini B, Sesti F, Piccione E. Office hysteroscopy: comparison of 2.7- and 4-mm hysteroscopes for acceptability, feasibility and diagnostic accuracy. J Reprod Med. 2005;50(1):45-8.

4. Litta P, Bonora M, Pozzan C, Merlin F, Sacco G, Fracas M, et al. Carbon dioxide versus normal saline for outpatient hysteroscopy. Hum Reprod. 2003; 18(1 1):2446-9.

5. Paschopoulos M, Kaponis A, Makrydimas G, Zikopoulos K, Alamanos $Y, O^{\prime}$ Donovan $P$, et al. Selecting distending medium for out-patient hysteroscopy. Does it really matter? Hum Reprod. 2004; 19(11):2619-25

6. Bettocchi S, Nappi L, Ceci O, Pontrelli G, Pinto L, Selvaggi L. Hysteroscopy and menopause: past and future. Curr Opin Obstet Gynecol. 2005;17(4):366-75.

7. Readman E, Maher PJ. Pain relief and outpatient hysteroscopy: a literature review. J Am Assoc Gynecol Laparosc. 2004; 11 (3):315-9.

8. Yang J, Vollenhoven B. Pain control in outpatient hysteroscopy. Obstet Gynecol Surv. 2002;57(10):693-702.

9. Al-Sunaidi $M$, Tulandi T. A randomized trial comparing local intracervical and combined local and paracervical anesthesia in outpatient hysteroscopy. J Minim Invasive Gynecol. 2007; 14(2):153-5.

10. Garbin O, Kutnahorsky R, Göllner JL, Vayssiere C. Vaginoscopic versus conventional approaches to outpatient diagnostic hysteroscopy: a two-centre randomized prospective study. Hum Reprod. 2006;21(11):2996-3000.

11. Crispi CP, Damian Júnior JC, Damian BB. Indicações. In: Crispi $P C$, editor. Tratado de videoendoscopia e cirurgia minimamente invasiva em ginecologia. $2 a$ ed. São Paulo: Revinter; 2006. p. 758-69.

12. Cicinelli E, Didonna T, Ambrosi G, Schönauer LM, Fiore $G$, Matteo MG. Topical anaesthesia for diagnostic hysteroscopy and endometrial biopsy in postmenopausal women: a randomised placebo-controlled double-blind study. Br J Obstet Gynaecol. 1997; 104(3):316-9.

13. Nagele F, Lockwood G, Magos AL. Randomised placebo controlled trial of mefenamic acid for premedication at outpatient hysteroscopy: a pilot study. Br J Obstet Gynaecol. 1997; 104(7):842-4.

14. da Costa AR, Pinto-Neto AM, Amorim M, Paiva LH, Scavuzzi A, Schettini J. Use of misoprostol prior to hysteroscopy in postmenopausal women: a randomized, placebo-controlled clinical trial. J Minim Invasive Gynecol. 2008;15(1):67-73.

15. Floris $S$, Piras B, Orrù M, Silvetti E, Tusconi A, Melis F. Efficacy of intravenous tramadol treatment for reducing pain during office diagnostic hysteroscopy. Fertil Steril. 2007;87(1):147-51.

16. Lin YH, Hwang JL, Huang LW, Chen HJ. Use of sublingual buprenorphine for pain relief in office hysteroscopy. J Minim Invasive Gynecol. 2005; 12(4):347-50.

17. Fernandez H, Alby JD, Tournoux C, Chauveaud-Lambling A, DeTayrac R, Frydman R, et al. Vaginal misoprostol for cervical ripening before operative hysteroscopy in pre-menopausal women: a double-blind, placebo-controlled trial with three dose regimens. Hum Reprod. 2004;19(7):1618-21.

18. Bettocchi S, Ceci O, Nappi L, Di Venere R, Masciopinto V, Pansini $V$, et al. Operative office hysteroscopy without anesthesia: analysis of 4863 cases performed with mechanical instruments. J Am Assoc Gynecol Laparosc. 2004;1 1 (1):59-61.

19. de Carvalho Schettini JA, Ramos de Amorim MM, Ribeiro Costa AA, Albuquerque Neto LC. Pain evaluation in outpatients undergoing diagnostic anesthesia-free hysteroscopy in a teaching hospital: a cohort study. J Minim Invasive Gynecol. 2007;14(6):729-35.

20. Lippi UG, Lopes RGC, Baracat FF. Ginecologia: manual de normas e condutas. São Paulo: EPUB; 2002. Histeroscopia.p. 23-33.

21. Bijur PE, Latimer CT, Gallagher EJ. Validation of a verbally administered numerical rating scale of acute pain for use in the emergency department. Acad Emerg Med. 2003;10(4):390-2.

22. Salo D, Eget D, Lavery RF, Garner L, Bernstein S, Tandon K. Can patients accurately read a visual analog pain scale? Am J Emerg Med. 2003;21 (7):515-9.

23. Sharma M, Taylor A, di Spiezio Sardo A, Buck L, Mastrogamvrakis $G$, Kosmas I, et al. Outpatient hysteroscopy: traditional versus the 'no-touch' technique. BJOG. 2005;1 12(7):963-7.

24. Cicinelli E, Schonauer LM, Barba B, Tartagni M, Luisi D, Di Naro E. Tolerability and cardiovascular complications of outpatient diagnostic minihysteroscopy compared with conventional hysteroscopy. J Am Assoc Gynecol Laparosc. 2003; 10(3):399-402.

25. Shankar M, Davidson A, Taub N, Habiba M. Randomised comparison of distension media for outpatient hysteroscopy. BJOG. 2004; 111 (1):57-62.

26. De Angelis $C, \operatorname{Re} M E$, Santoro G. Pelvic pain, low blood pressure, and hemolysis after outpatient hysteroscopy in a patient with glucose-6-phosphate dehydrogenase deficiency. Fertil Steril. 2003;79(6): 1442-3.

27. De Angelis C, Santoro G, Re ME, Nofroni I. Office hysteroscopy and compliance: mini-hysteroscopy versus traditional hysteroscopy in a randomized trial. Hum Reprod. 2003;18(11):2441-5

28. Almeida ZMMCA, Pontes R, Costa HLFF. Avaliação da dor na histeroscopia diagnóstica por vaginoscopia utilizando-se, como meio de distensão, solução salina à temperatura corporal: ensaio clínico randomizado. Rev Bras Ginecol Obstet. 2008;30(1):25-30.

29. Bettocchi S, Selvaggi L. A vaginoscopic approach to reduce the pain of office hysteroscopy. J Am Assoc Gynecol Laparosc. 1997;4(2):255-8.

30. Lau WC, Ho RY, Tsang MK, Yuen PM. Patient's acceptance of outpatient hysteroscopy. Gynecol Obstet Invest. 1999;47(3):191-3.

31. De laco P, Marabini A, Stefanetti M, Del Vecchio C, Bovicelli L. Acceptability and pain of outpatient hysteroscopy. J Am Assoc Gynecol Laparosc. 2000;7(1):71-5.

32. Sharma JB, Aruna J, Kumar P, Roy KK, Malhotra N, Kumar S. Comparison of efficacy of oral drotaverine plus mefenamic acid with paracervical block and with intravenous sedation for pain relief during hysteroscopy and endometrial biopsy. Indian J Med Sci. 2009;63(6):244-52.

33. Singh N, Ghosh B, Naha M, Mittal S. Vaginal misoprostol for cervical priming prior to diagnostic hysteroscopy- efficacy, safety and patient satisfaction: a randomized controlled trial. Arch Gynecol Obstet. 2009;279(1):37-40. 
34. Morgan M, Dodds W, Wolfe C, Raju S. Women's views and experiences of outpatient hysteroscopy: implications for a patientcentered service. Nurs Health Sci. 2004;6(4):315-20.

35. Campo R, Molinas CR, Rombauts L, Mestdagh G, Lauwers $M$, Braekmans $P$, et al. Prospective multicentre randomized controlled trial to evaluate factors influencing the success rate of office diagnostic hysteroscopy. Hum Reprod. 2005;20(1): 258-63.

36. Sagiv R, Sadan O, Boaz M, Dishi M, Schechter E, Golan A. A new approach to office hysteroscopy compared with traditional hysteroscopy: a randomized controlled trial. Obstet Gynecol. 2006; 108(2):387-92.

37. Guida M, Di Spiezio Sardo A, Acunzo G, Sparice S, Bramante $S$, Piccoli $R$, et al. Vaginoscopic versus traditional office hysteroscopy: a randomized controlled study. Hum Reprod. 2006;21(12):3253-7.

38. Madari S, Al-Shabibi, Papalampros P, Papadimitriou A, Magos A. A randomised trial comparing the $\mathrm{H}$ Pipelle with the standard Pipelle for endometrial sampling at 'no-touch' (vaginoscopic) hysteroscopy. BJOG. 2009;1 16(1):32-7. 